\title{
LncRNA SNHG12 regulates gastric cancer progression by acting as a molecular sponge of miR-320
}

\author{
HANYUN ZHANG ${ }^{1,2}$ and WENJIE LU ${ }^{2}$ \\ Departments of ${ }^{1}$ Gastroenterology and ${ }^{2}$ General Surgery, The Second Affiliated Hospital of Zhejiang University, \\ Medical College, Hangzhou, Zhejiang 310009, P.R. China
}

Received July 25, 2017; Accepted October 4, 2017

DOI: $10.3892 / \mathrm{mmr} .2017 .8143$

\begin{abstract}
Gastric cancer (GC) is one of the most common malignancies worldwide. Previous studies have focused on long non-coding RNAs (lncRNAs), which have important roles in the development and progression of GC. The present study aimed to clarify the expression and function of lncRNA small nucleolar RNA host gene 12 (SNHG12) in GC. The expression and the clinical characteristics of GC were analyzed in the samples from patients with GC and matched adjacent normal tissues. The present study determined that SNHG12 was significantly overexpressed in GC and its expression level was highly associated with tumor size, tumor-node-metastasis stage, distant metastasis, lymphatic metastasis. Patients with high SNHG12 expression had a short survival period. Additionally, inhibition of SNHG12 in GC cell lines SGC-7901 and AGS suppressed cell growth, colony formation, proliferation and invasion. MicroRNA (miR)-320, a putative target gene of SNHG12, was inversely correlated with SNHG12 expression in GC tissues and cell lines. In addition, the present study determined that miR-320 was directly regulated by SNHG12 and suppression of miR-320 expression reversed the inhibitory effects of SNHG12 siRNA on $\mathrm{GC}$ cell proliferation and invasion. These findings revealed that SNHG12 acts as a tumor promoter by directly targeting miR-320 in GC, suggesting a potential novel biomarker for the diagnosis and prognosis of GC.
\end{abstract}

\section{Introduction}

Gastric cancer (GC), as a common malignancy of digestive tract carcinomas, is one of the leading causes of cancer-associated mortality worldwide, and it has been previously reported that from $>950,000$ new GC cases, 720,000 deaths occurred

Correspondence to: Dr Wenjie Lu, Department of General Surgery, The Second Affiliated Hospital of Zhejiang University, Medical College, 88 Jiefang Road, Hangzhou, Zhejiang 310009, P.R. China

E-mail: luwenjie@zju.edu.cn

Key words: SNHG12, gastric cancer, miR-320, proliferation, invasion in annually (1-3). Despite the early diagnosis and effective therapies, GC is often diagnosed at advanced stage with the characters of extensive invasion and distant metastasis, leading to the low survival rate $(4,5)$. Additionally, as it is a major health threat, GC puts a large economic burden on every patient's family (5). Therefore, there is an urgency to identify novel biomarkers that can be detected early, and at the same time they can block or slow down the malignancy progression in order to improve GC prognosis. Previous studies have identified various cellular products to be novel molecules or targets in the progression of GC, including long non-coding RNAs (lncRNAs) $(6,7)$.

LncRNAs are identified as a class RNA molecules with length of more than 200 nucleotides, that have no significant protein-coding capacity, but occupy a majority of mammalian genome production $(8,9)$. LncRNAs are involved in various biological processes including cell proliferation, apoptosis and migration (10). Additionally, lncRNAs have been reported to function as important genes in human diseases, including lncRNA PVT1 was demonstrated to be associated with prostate cancer and H19 was associated with breast cancer (11-13). Recent studies have revealed that lncRNAs also have critical roles in the progression and metastasis of GC $(14,15)$. However, the molecular mechanism of lncRNAs in the progression and metastasis of GC remains to be elucidated. Identification of novel biomarkers is required for the diagnosis and treatment of patients with GC.

Small nucleolar RNA host gene 12 (SNHG12) is located in chromosome 1 and 675 nucleotides in size. Previous studies suggested that SNHG12 exerted its oncogenic function by promoting cell growth and inhibiting cell apoptosis in various cancer types $(10,16)$. The present study aimed to investigate the role of SNHG12 in GC. Therefore, SNHG12 expression and its clinicopathological and prognostic significance for GC patients was assessed. Subsequently, the function of SNHG12 on the proliferation and invasion of GC cell lines was examined. The findings of the current study suggested that SNHG12 may be a tumor promoter by acting as a miR-320 sponge in GC, indicating that SNHG12 may be a novel biomarker for GC progression.

\section{Materials and methods}

Human tissue specimens and cell culture. GC tissues and adjacent normal gastric epithelial tissues were obtained from 
60 patients who underwent surgery at Department of General Surgery, Second Affiliated Hospital of Zhejiang University (Hangzhou, China) between May 2015 and June 2016. Specimens were immediately frozen in liquid nitrogen and stored at $-80^{\circ} \mathrm{C}$. Informed consent was obtained from all patients. The present study was approved by the Ethics Committee of the Second Affiliated Hospital of Zhejiang University. The clinical characteristics recorded included sex, age, size of carcinoma, tumor-node-metastasis (TNM) stage, distant and lymphatic metastasis. Overall survival and the disease-free survival rates were recorded from the date of surgery to the mortality of the patient or last contact.

The SGC7901, NCI-N87 and AGS human gastric cancer cell lines and normal gastric epithelial cell line GES-1 were obtained from American Type Culture Collection (Manassas, VA, USA). Cells were cultured in Dulbecco's modified Eagle's medium (cat. no. sc-224478; Santa Cruz Biotechnology, Inc., Dallas, TX, USA) supplemented $10 \%$ fetal bovine serum (FBS), 100 U/ml penicillin (cat. no. sc-391048; Santa Cruz Biotechnology, Inc.) and $100 \mathrm{U} / \mathrm{ml}$ streptomycin (cat. no. sc-202821; Santa Cruz Biotechnology, Inc.) at $37^{\circ} \mathrm{C}$ in a humidified $5 \% \mathrm{CO}_{2}$ atmosphere.

Reverse transcription-quantitative polymerase chain reaction $(R T-q P C R)$. Total RNA was extracted from cells or tissues using TRIzol (Invitrogen; Thermo Fisher Scientific, Inc., Waltham, MA, USA). cDNA synthesis was performed at $37^{\circ} \mathrm{C}$ for $15 \mathrm{~min}$, then $85^{\circ} \mathrm{C}$ for $5 \mathrm{sec}$ using reverse transcriptase (Applied Biosystems; Thermo Fisher Scientific, Inc.) following the manufacturer protocol. qPCR was conducted with ABI 7500 system (Applied Biosystems; Thermo Fisher Scientific, Inc.) using SYBR-Green (Takara Biotechnology Co., Ltd., Dalian, China). PCR was performed for 25 cycles of: $10 \mathrm{sec}$ at $98^{\circ} \mathrm{C}, 10 \mathrm{sec}$ at $55^{\circ} \mathrm{C}$ and $20 \mathrm{sec}$ at $72^{\circ} \mathrm{C}$. The primer sequences used were as follows: SNHG12: Forward (F), 5'-TCTGGTGATCGAGGACTTCC-3' and reverse (R), 5'-ACC TCCTCAGTATCACACACT-3'; GAPDH F, 5'-ACACCCACT CCTCCACCTTT-3' and R, 5'-TTACTCCTTGGAGGCCA TGT-3'. LncRNA, mRNA and miRNA expression levels were normalized using U6 small RNA. Fold-changes were counted using the $2^{-\Delta \Delta \mathrm{Cq}}$ method (17).

Cell transfection. SNHG12 siRNA (si-SNHG12; 5'-GCA GTGGCGTGATCA AGGCTCATTGCAGCCT-3') and control siRNA (si-ctrl; 5'-TTCCTGCGTGGGCCCAGC GCCGGGCACTGA-3') were purchased from Guangzhou RiboBio Co., Ltd. (Guangzhou, China). miR-320 and control miRNA mimic/inhibitors were synthesized by GenePharma Co., Ltd., (Shanghai, China). Transfections were performed using a Lipofectamine 2000 kit (Invitrogen; Thermo Fisher Scientific, Inc.) according to the manufacturer instructions. The transfected cells were harvested and assessed $48 \mathrm{~h}$ post-transfection.

Cell proliferation assays. Cell viability was determined using Cell Counting Kit-8 (CCK-8) and the colony formation assays. For the CCK- 8 assay, the transfected GC cells $\left(2 \times 10^{3}\right)$ were seeded into 96 -well plates and incubated for 1-6 days at $37^{\circ} \mathrm{C}$. CCK-8 (10 $\mu$; Beyotime Institute of Biotechnology, Shanghai, China) solution was added to each well $1 \mathrm{~h}$ prior to the endpoint.
Subsequently, the plates were incubated at $37^{\circ} \mathrm{C}$ for $2 \mathrm{~h}$. A Benchmark Microplate Reader (Bio-Rad Laboratories, Inc., Hercules, CA, USA) was used to determine the spectrophotometric absorbance value at $460 \mathrm{~nm}$. For the colony formation assay, the transfected cells (500 cells/well) were placed into 6-well plates and cultured in RPMI 1640 medium (Gibco; Thermo Fisher Scientific, Inc.) containing $10 \% \mathrm{FBS}$ at $37^{\circ} \mathrm{C}$ with $5 \% \mathrm{CO}_{2}$ for 10 days. Rinsed with phosphate-buffered saline (PBS), the cells were fixed with $4 \%$ paraformaldehyde for $15 \mathrm{~min}$ at room temperature and stained with crystal violet $(1 \mathrm{mg} / \mathrm{ml})$ at $37^{\circ} \mathrm{C}$ for $10 \mathrm{~min}$. A total of ten fields were selected at random to examine and the number of colonies ( $\geq 50$ cells) was detected with a light microscope.

Immunohistochemistry (IHC) analysis. IHC was conducted as previously described (18). Briefly, the transfected cells were grown on coverslips and fixed with $4 \%$ paraformaldehyde for $30 \mathrm{~min}$ at room temperature. Following being rinsed three times with 0.1 M PBS ( $\mathrm{pH} 7.4$ ), the cells were incubated with a primary mouse anti-Ki67 antibody (cat. no. 612254; 1:250; BD Biosciences, Franklin Lakes, NJ, USA) overnight at $4^{\circ} \mathrm{C}$. The next day, the cells were incubated with rabbit horseradish peroxidase (HRP-conjugated secondary antibody IgG (cat. no. sc-3749, 1:500; Santa Cruz Biotechnology, Inc.) for $15 \mathrm{~min}$ at room temperature, followed by 3,3'-diaminobenzidine staining at room temperature for $4 \mathrm{~min}$ and counterstained with hematoxylin. Finally, the cells were assessed using a light microscope.

Invasion assay. Cell invasive capacity was determined using an invasion assay. The transfected cells $\left(2 \times 10^{5}\right)$ were seeded in the top chamber with Matrigel-coated membrane (24-well insert; pore size: $8-\mu \mathrm{m}$; Corning Inc., Corning, NY, USA) of the inserts. The RPMI 1640 media with 10\% FBS was added to the bottom chamber. The cells were incubated for $48 \mathrm{~h}$, the cells on the upper surface of the membrane were swabbed off gently, and cells on the lower surface were fixed in $4 \%$ paraformaldehyde for $15 \mathrm{~min}$ at room temperature and stained with $2 \%$ crystal violet at $37^{\circ} \mathrm{C}$ for $10 \mathrm{~min}$ (Sigma-Aldrich; Merck Millipore, Darmstadt, Germany) staining. Total number of invasive cells were counted using a light microscope BX51 (Olympus America, Inc., Melville, NY, USA).

Western blot assay. Total protein $(50 \mu \mathrm{g})$ was extracted using radioimmunoprecipitation assay lysis buffer (Sigma-Aldrich; Merck $\mathrm{KGaA}$ ) and quantified by bicinchoninic acid assay kit (Beyotime Institute of Biotechnology). Then the protein was separated using $10 \%$ SDS-PAGE and transferred onto polyvinylidene fluoride membranes. The membranes were immunoblotted with $5 \%$ powdered milk dissolved in PBS containing $0.1 \%$ Tween-20 at room temperature for $3 \mathrm{~h}$. Then the membranes were incubated overnight at $4^{\circ} \mathrm{C}$ with primary rabbit antibodies: CRK like proto-oncogene, adaptor protein (CRKL; 1:500; cat. no. sc-365471; Santa Cruz Biotechnology, Inc.), phosphorylated (p)-AKT serine/threonine kinase 1 (Akt; 1:500; cat. no. sc-24500; Santa Cruz Biotechnology, Inc.), p-extracellular-signal regulated kinase (Erk; 1:1,000; cat. no. 4370; Cell Signaling Technology, Inc., Danvers, MA, USA) and GAPDH (1:2,000; cat. no. sc-420485; Santa Cruz Biotechnology, Inc.). Then, a 1:3,000 HRP-conjugated anti-rabbit secondary antibody 
A
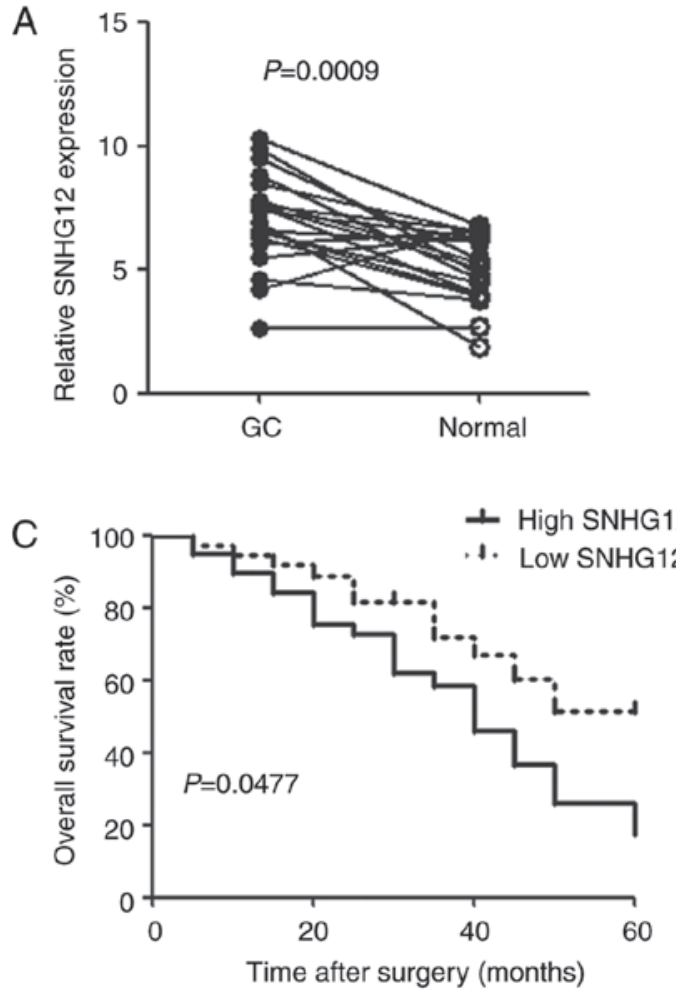

B

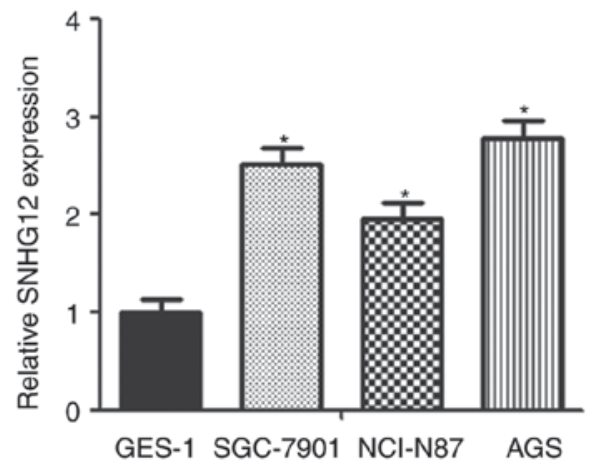

D

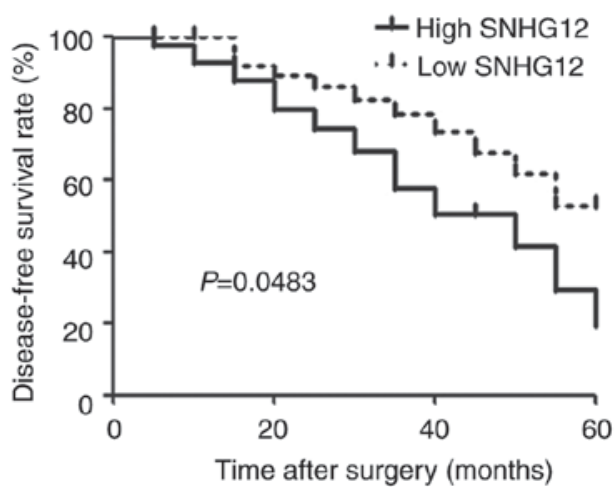

Figure 1. Prognostic value of SNHG12 in GC specimens. SNHG12 expression was detected in (A) GC tissues ( $\mathrm{n}=20)$ and (B) cell lines (n=5) by reverse transcription-quantitative polymerase chain reaction. ${ }^{*} \mathrm{P}<0.05$ vs. control group. Association between SNHG12 expression and (C) overall survival rate and (D) disease-free survival rate of $60 \mathrm{GC}$ patients were determined by Kaplan-Meier analysis. For each cohort, sub-groups were divided according to the cut-off values of SNHG12 and GC. SNHG12, small nucleolar RNA host gene 12; GC, gastric cancer.

(cat. no. 1662,408; 1:3,000; Bio-Rad Laboratories Inc., Hercules, CA, USA) was incubated with the membrane for $1 \mathrm{~h}$ at room temperature. Signals were quantified using a ChemiDoc XRS system (Bio-Rad Laboratories, Inc.).

Reporter vector construction and luciferase reporter assay. A bioinformatics database (lncipedia.org) was used to search for potential microRNAs that may bind to SNHG12. It was demonstrated that SNHG12 fragments contained the predicted binding site of miR-320. Subsequently, the fragments were amplified using PCR (17) and cloned them into pGL3-control vector (Promega Corporation, Madison, WI, USA) to form the reporter vector SNHG12-wild-type (Wt SNHG12). To test the binding specificity, the corresponding mutant fragments were cloned to form the reporter vector SNHG12-mutant-type (Mut SNHG12). SGC7901 and AGS cells (1.0x10 6 cells) were seeded into a 6-well dish with reporter vector Wt SNHG12 or Mut SNHG12 (100 ng) and indicated miRNAs (25 nM) using Lipofectamine 2000 (Invitrogen; Thermo Fisher Scientific, Inc.). Each well had $800 \mathrm{ml}$ serum-free medium added and cells were incubated for $6 \mathrm{~h}$ in a $\mathrm{CO}_{2}$-incubator. Luciferase activity was detected $48 \mathrm{~h}$ after co-transfection using a dual-luciferase reporter assay system normalized by Renilla luciferase (Promega Corporation, Madison, WI, USA) following the manufacturer's protocol.

Statistical analysis. Data are presented as the mean \pm standard deviation and statistical analysis was performed using SPSS version 18.0 (SPSS, Inc., Chicago, IL, USA). Differences between groups were determined using Student's t-test and one-way analysis of variance (ANOVA). Tukey's test was used when it was necessary following a one-way ANOVA. Survival curves were analyzed using the Kaplan-Meier method and calculated using the log-rank test. The relationship of SNHG12 and miR-320 expression was detected using Pearson correlation. $\mathrm{P}<0.05$ was considered to indicate a statistically significant difference.

\section{Results}

SNHG12 is upregulated in GC tissues and cell lines. The expression of SNHG12 was examined in GC tissues and adjacent normal tissues. According to the RT-qPCR analysis, the expression of SNHG12 was higher in GC tissues compared with normal tissues (Fig. 1A). SNHG12 expression increased significantly in gastric cancer cell lines, including SGC7901, NCI-N87 and AGS compared with normal gastric epithelial cells GES-1 (Fig. 1B).

Correlations between SNHG12 expression in patients with GC and clinical characteristics. To investigate the relationship of clinical characteristics and SNHG12 expression in gastric cancer, a cut-off value (median expression value) of SNHG12 expression was used to divide all 60 patients into two groups. As presented in Table I, patients with SNHG12 level higher than the cut-off value were placed in the high expression group, and patients with lower SNHG12 expression were assigned to the low expression group. The expression of SNHG12 was positively associated with tumor size $(\mathrm{P}=0.0043)$, TNM stage $(\mathrm{P}=0.0384)$, distant metastasis $(\mathrm{P}=0.0018)$ and lymphatic 
Table I. Correlations between SNHG12 expression in patients with gastric cancer and their clinical characteristics.

\begin{tabular}{|c|c|c|c|c|}
\hline \multirow[b]{2}{*}{ Group } & \multirow[b]{2}{*}{ Number of patients } & \multicolumn{2}{|c|}{$\begin{array}{c}\text { Relative } \\
\text { SNHG12 expression }\end{array}$} & \multirow[b]{2}{*}{ P-value } \\
\hline & & Low & High & \\
\hline Sex & & & & 0.7952 \\
\hline Male & 34 & 17 & 16 & \\
\hline Female & 26 & 13 & 14 & \\
\hline Age & & & & 0.7906 \\
\hline$>50$ & 38 & 11 & 12 & \\
\hline$\leqq 50$ & 22 & 19 & 18 & \\
\hline Tumor size $(\mathrm{cm})$ & & & & 0.0043 \\
\hline$>5$ & 19 & 11 & 22 & \\
\hline$\leqq 5$ & 41 & 19 & 8 & \\
\hline TNM stage & & & & 0.0384 \\
\hline I-II & 28 & 18 & 10 & \\
\hline III-IV & 32 & 12 & 20 & \\
\hline Distant metastasis & & & & 0.0018 \\
\hline Negative & 27 & 19 & 7 & \\
\hline Positive & 33 & 11 & 23 & \\
\hline Lymphatic metastasis & & & & 0.0045 \\
\hline No & 36 & 21 & 10 & \\
\hline Yes & 24 & 9 & 20 & \\
\hline
\end{tabular}

SNHG12, small nucleolar RNA host gene 12; TNM, tumor node metastasis.

metastasis $(\mathrm{P}=0.0045)$. However, no significant difference was observed between SNHG12 level and sex or age. Patients with high SNHG12 expression presented lower overall survival rate and disease-free survival rate compared with those with low SNHG12 expression according to the Kaplan-Meier analysis $(\mathrm{P}=0.0477, \mathrm{P}=0.0483$, respectively; Fig. $1 \mathrm{C}$ and $\mathrm{D})$.

Knockdown of SNHG12 inhibits cell proliferation and invasion in vitro. To elucidate the biological function of SNHG12, the present study downregulated SNHG12 in GC cells by transfection with SNHG12 siRNA. RT-qPCR was used to detect SNHG12 expression in transfected cells (Fig. 2A). CCK-8 assay demonstrated that downregulation of SNHG12 suppressed cell growth (Fig. 2B). The colony formation assay revealed that the number of colonies decreased significantly in the SNHG12 knockdown group compared with the control group (Fig. 2C). Ki67-labeled nuclei were reduced in cells transfected with si-SNHG12 compared with cells transfected with si-ctrl according to IHC analysis (Fig. 2D). In addition, cell invasion was markedly inhibited in cells with SNHG12 knockdown compared with cells transfected with si-ctrl (Fig. 2E).

SNHG12 promotes cell proliferation and invasion by targeting miR-320 directly. A previous study identified lncRNAs as competitive endogenous RNA (ceRNA) for miRNAs or naturally occurring miRNA sponges, which have a critical function in the progression of gene expression and signaling pathways (19). The present study used LNCipedia (Incipedia. org) to predict putative miRNA, which is modulated by SNHG12 and determined that miR-320 was the potential target of SNHG12. To clarify whether there were direct interactions between SNHG12 and miR-320, the correlation between the expression of SNHG12 and miR-320 in GC tissue specimens was investigated. A negative correlation was observed between SNHG12 and miR-320 (Fig. 3A). The relative miR-320 expression increased significantly after the knockdown of SNHG12 in GC cell lines (Fig. 3B). Additionally, western blot analysis demonstrated that the expression of target genes (CRKL, p-Akt and p-Erk) of miR-320 was downregulated after the knockdown of SNHG12 (Fig. 3C).

To determine whether SNHG12 may directly bind miR-320 in GC cells, the present study cloned the Wt and Mut SNHG12 sequences, which contained the potential binding site of miR-320 into a luciferase reporter gene system (Fig. 3D). As expected, miR-320 in both GC cell lines led to a significantly reduced luciferase activity of SNHG12, containing a Wt sequence without suppressing the activity of SNHG12 with a mutant sequence (Fig. 3E).

Inhibition of miR-320 reverses SNHG12-induced effects on $G C$ cells. In order to determine whether SNHG12 exerts its function by sponging miR-320, the SNHG12-downregulated GC cells were transfected with $\mathrm{miR}-320 / \mathrm{miR}$-con inhibitor (Fig. 4A). The CCK-8 assay demonstrated that cell growth was significantly promoted in the miR-320 inhibitor group (Fig. 4B). Similarly, IHC analysis revealed that transfection 

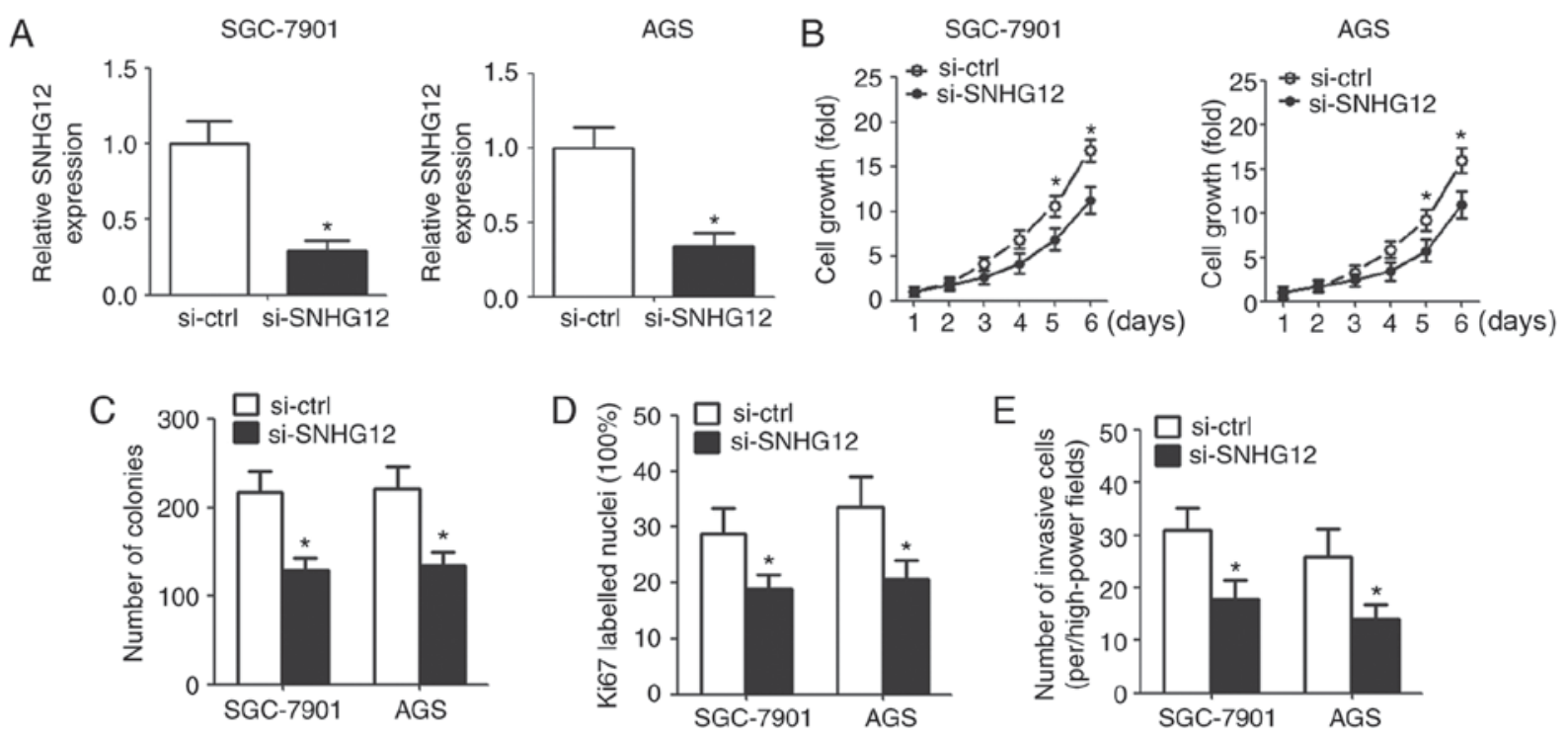

Figure 2. Effects of SNHG12 on GC cell proliferation and invasion. SGC-7901 and AGS cells were transfected with si-SNHG12 and si-ctrl for 48 h. (A) Knockdown of SNHG12 in GC (SGC-7901 and AGS) cells by reverse transcription-quantitative polymerase chain reaction. (B) Cell Counting Kit-8 assays were conducted to determine cell viability at indicated time points. (C) Number of colonies were examined after transfection using colony formation assay. (D) Immunohistochemistry was performed to detect the Ki67-labeled nuclei. (E) Number of invasive cells was analyzed using invasion assays. Data are expressed as the mean \pm standard deviation, $n=5$. "P<0.05 vs. control group. GC, gastric cancer; SNHG12, small nucleolar RNA host gene 12; si-SNHG12, SNHG12 small interfering RNA; si-ctrl, control small interfering RNA; Ki67, antigen identified by monoclonal antibody Ki 67.
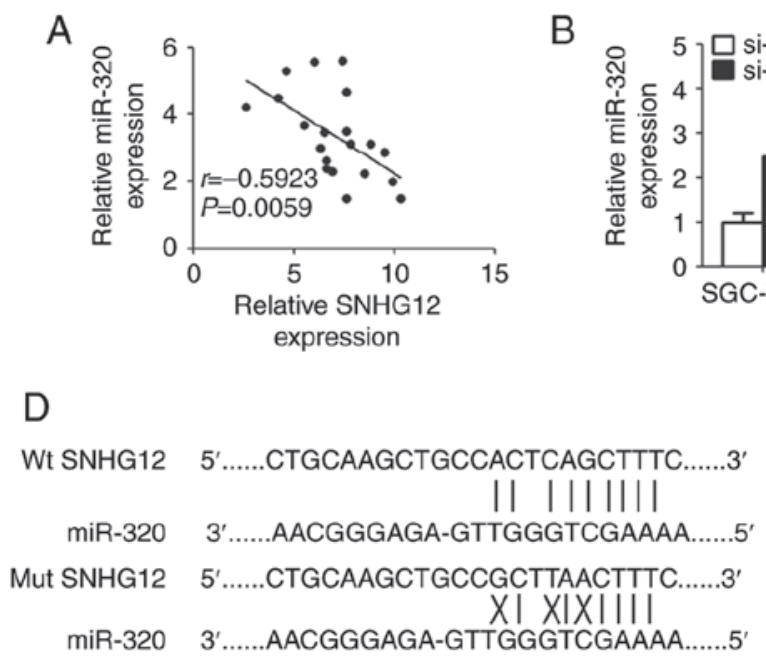

B

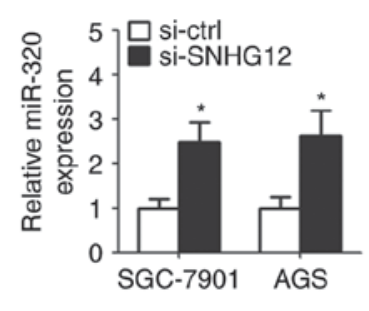

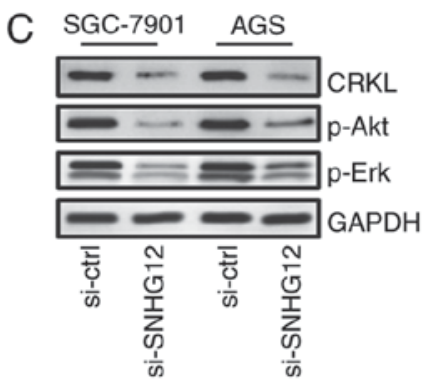

$\mathrm{E}$
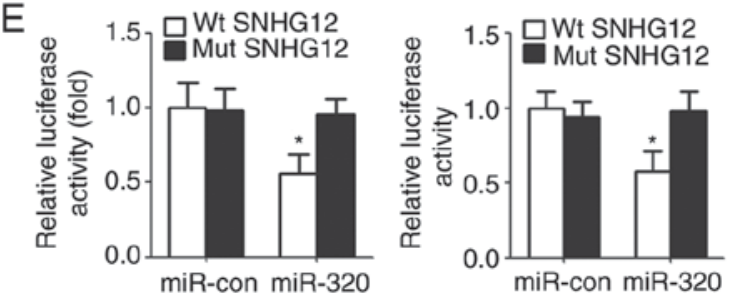

Figure 3. SNHG12 may directly target miR-320. (A) Inverse relationship between the SNHG12 and miR-320 expression levels was identified in GC tissues. (B) Upregulation of miR-320 was found in GC cells with the knockdown of SNHG12 by reverse transcription-quantitative polymerase chain reaction. (C) Western blot analysis of CRKL, p-Akt and p-Erk expression in SGC-7901 and AGS cells $48 \mathrm{~h}$ post-transfection. (D) Putative SNHG12 target sequences in miR-320 are presented. (E) SGC-7901 and AGS cells were co-transfected with the luciferase reporter plasmid carrying the Wt/Mut sequences of SNHG12 and control or miR-320 mimics. A dual-luciferase reporter system analysis was performed. Data were expressed as the mean \pm standard deviation, $\mathrm{n}=5$. ${ }^{*} \mathrm{P}<0.05 \mathrm{vs}$. control group. miR-320, microRNA-320; miR-con, microRNA control; SNHG12, small nucleolar RNA host gene 12; si-SNHG12, SNHG12 small interfering RNA; si-ctrl, control small interfering RNA; Wt, wild-type; Mut, mutant; CRKL, CRK like proto-oncogene, adaptor protein; p-Akt, phosphorylated-AKT serine/threonine kinase 1; p-Erk, phosphorylated-extracellular-signal regulated kinase.

with the miR-320 inhibitor increased Ki67-labeled nuclei in SNHG12-downregulated GC cells (Fig. 4C). The number of colonies and invasive cells were also upregulated in the miR-320 knockdown cells (Fig. 4D and E).

\section{Discussion}

GC is a common disease with difficult complications that include metastasis and recurrence, which seriously affect the survival rate of patients with GC (20). A previous study revealed novel functions of lncRNAs in the development and metastasis of human cancers (21). SNHG12, being one of the lncRNAs, with the small nucleolus RNA-encoding sequences in its introns has been reported to contribute to the progression of various tumors, such as hepatocellular carcinoma and osteosarcoma $(16,22)$. To the best of the authors' knowledge; however, the function of SNHG12 in GC has not been previously explored. 
A

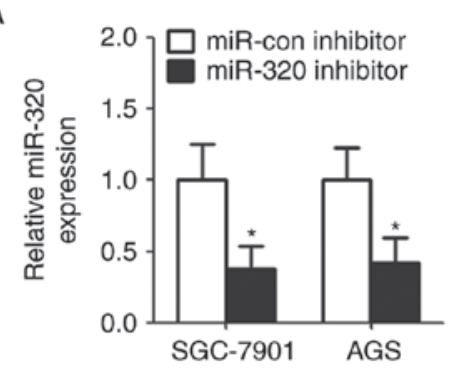

C

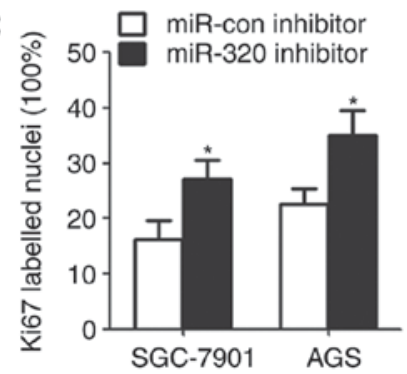

B
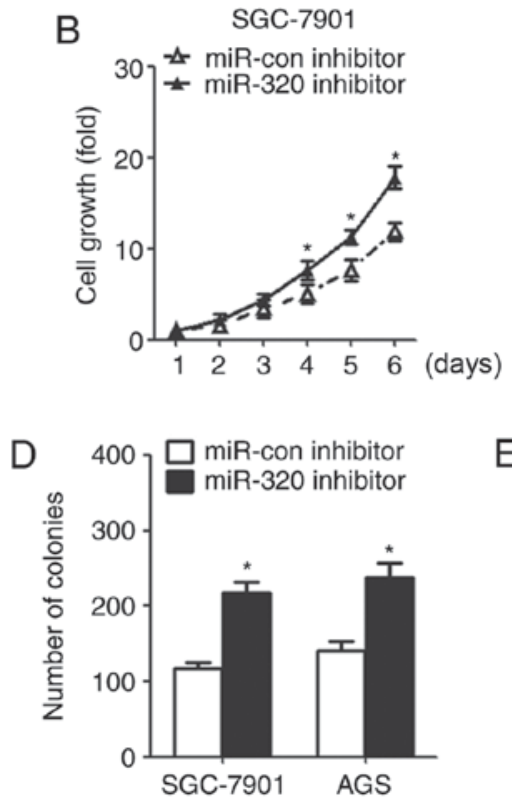

AGS

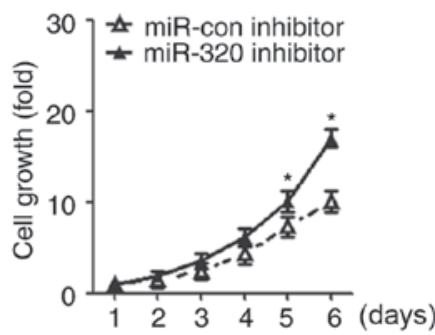

$\mathrm{E}$

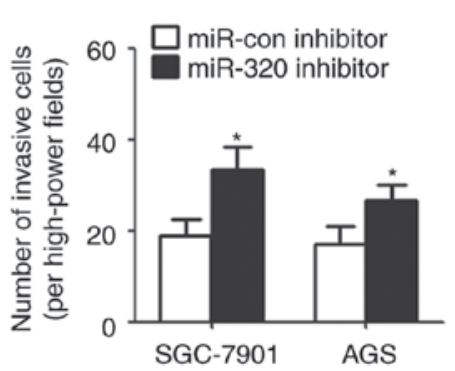

Figure 4. Downregulation of miR-320 reversed the effect of SNHG12 siRNA. SGC-7901 and AGS cells were co-transfected with the si-SNHG12 and miR-320/miR-con inhibitor for $48 \mathrm{~h}$. (A) Expression of miR-320 was analyzed by reverse transcription-quantitative polymerase chain reaction. (B) Cell growth curves for GC cells were determined using Cell Counting Kit-8 assay. (C) Ki67-labeled nuclei for GC cells were quantified using immunohistochemistry assay. (D) Number of colonies were examined by colony formation assays. (E) Number of invasive cells was detected by invasion assay. Data are expressed as the mean \pm standard deviation, $\mathrm{n}=5$. ${ }^{*} \mathrm{P}<0.05$ vs. control group. miR-320, microRNA-320; miR-con, microRNA control; SNHG12, small nucleolar RNA host gene 12; si-SNHG12, SNHG12 small interfering RNA; miR-con, micro RNA control.

The present study determined that the expression level of SNHG12 was significantly upregulated in GC tissues and SGC-7901, NCI-N87 and AGS GC cell lines. Additionally, the expression of SNHG12 was closely associated with tumor size, TNM stage, distant and lymphatic metastasis. In addition, patients with high SNHG12 expression had a lower overall survival and disease-free survival rates according to the 60-month follow-up survival survey. Additionally, silencing of SNHG12 in GC cells inhibited cell proliferation and invasion. These findings suggested that SNHG12 may be involved in the carcinogenesis and progression of GC and it may be a prognostic marker for predicting overall and disease-free survival of patients with GC. These findings were consistent with previous studies demonstrating that SNHG12 acts as a promoter in carcinoma progression and development $(23,24)$.

Additionally, the present study validated miR-320 as a direct-target gene of SNHG12, and identified a negative correlation between SNHG12 and miR-320. Based on the bioinformatics analysis, the putative binding sites between SNHG12 and miR-320 were revealed. Dual luciferase assay determined that miR-320 was a direct downstream target of SNHG12. These findings suggested that miR-320, a member of the miR-200 family, may have an essential role in the progression of GC. This finding is consistent with previous studies $(25,26)$. miR-320 has been previously reported to be implicated in many cancer-associated biological processes, such as cell proliferation, invasion and metastasis (27-29). A previous study indicated that miR-320 may inhibit proliferation and invasion by targeting CRKL through the ERK and AKT pathway (30). SNHG12 was observed to upregulate the CRKL (a target gene of miR-320) expression in the present study, which subsequently affected AKT and ERK signaling. In addition, it was determined that the inhibition of miR-320 reversed the pro-tumor effects of SNHG12 on cell proliferation, colony formation and invasion of GC cells.

In conclusion, the present study demonstrated that SNHG12 may act as a promoter of tumorigenesis and development of GC and become a potential prognostic biomarker for the diagnosis and prognosis of GC. Additionally, SNHG12 was confirmed to act as a sponge for miR-320 to modulate the CRKL expression, and further affected the downstream AKT and ERK pathways.

\section{Acknowledgements}

The present study was supported by Zhejiang Public Welfare Technology Research and Social Development Project (grant no. 2014C33187).

\section{References}

1. Gu J, Li Y, Fan L, Zhao Q, Tan B, Hua K and Wu G: Identification of aberrantly expressed long non-coding RNAs in stomach adenocarcinoma. Oncotarget 8: 49201-49216, 2017.

2. Siegel R, Naishadham D and Jemal A: Cancer statistics, 2012. CA Cancer J Clin 62: 10-29, 2012.

3. Torre LA, Bray F, Siegel RL, Ferlay J, Lortet-Tieulent J and Jemal A: Global cancer statistics, 2012. CA Cancer J Clin 65: 87-108, 2015.

4. Zhang G, Zhao X, Li J, Yuan Y, Wen M, Hao X, Li P and Zhang A: Racial disparities in stage-specific gastric cancer: Analysis of results from the Surveillance Epidemiology and End Results (SEER) program database. J Investig Med 65: 991-998, 2017.

5. Chen DL, Ju HQ, Lu YX, Chen LZ, Zeng ZL, Zhang DS, Luo HY, Wang F, Qiu MZ, Wang DS, et al: Long non-coding RNA XIST regulates gastric cancer progression by acting as a molecular sponge of miR-101 to modulate EZH2 expression. J Exp Clin Cancer Res 35: 142, 2016.

6. Liu Z, Chen Z, Fan R, Jiang B, Chen X, Chen Q, Nie F, Lu K and Sun M: Over-expressed long noncoding RNA HOXA11-AS promotes cell cycle progression and metastasis in gastric cancer. Mol Cancer 16: 82, 2017. 
7. Li J, Gao J, Tian W, Li Y and Zhang J: Long non-coding RNA MALAT1 drives gastric cancer progression by regulating HMGB2 modulating the miR-1297. Cancer Cell Int 17: 44, 2017.

8. Wang KC and Chang HY: Molecular mechanisms of long noncoding RNAs. Mol Cell 43: 904-914, 2011.

9. Wapinski $\mathrm{O}$ and Chang HY: Long noncoding RNAs and human disease. Trends Cell Biol 21: 354-361, 2011.

10. Wang O, Yang F, Liu Y, Lv L, Ma R, Chen C, Wang J, Tan Q, Cheng Y, Xia E, et al: C-MYC-induced upregulation of lncRNA SNHG12 regulates cell proliferation, apoptosis and migration in triple-negative breast cancer. Am J Transl Res 9: 533-545, 2017.

11. Troy A and Sharpless NE: Genetic 'Inc'-age of noncoding RNAs to human disease. J Clin Invest 122: 3837-3840, 2012.

12. Zhang Y, Xu Y, Feng L, Li F, Sun Z, Wu T, Shi X, Li J and Li X: Comprehensive characterization of lncRNA-mRNA related ceRNA network across 12 major cancers. Oncotarget 7: 64148-64167, 2016.

13. Ning S, Gao Y, Wang P, Li X, Zhi H, Zhang Y, Liu Y, Zhang J, Guo M, Han D and Li X: Construction of a lncRNA-mediated feed-forward loop network reveals global topological features and prognostic motifs in human cancers. Oncotarget 7: 45937-45947, 2016.

14. Zhang F, Zhang $L$ and Zhang C: Long noncoding RNAs and tumorigenesis: Genetic associations, molecular mechanisms, and therapeutic strategies. Tumour Biol 37: 163-175, 2016.

15. Huang B, Song JH, Cheng Y, Abraham JM, Ibrahim S, Sun Z, $\mathrm{Ke} X$ and Meltzer SJ: Long non-coding antisense RNA KRT7-AS is activated in gastric cancers and supports cancer cell progression by increasing KRT7 expression. Oncogene 35: 4927-4936, 2016.

16. Wang JZ, Xu CL, Wu H and Shen SJ: LncRNA SNHG12 promotes cell growth and inhibits cell apoptosis in colorectal cancer cells. Braz J Med Biol Res 50: e6079, 2017.

17. Livak KJ and Schmittgen TD: Analysis of relative gene expression data using real-time quantitative PCR and the 2(-Delta Delta C(T)) method. Methods 25: 402-408, 2001

18. Singh RP, Deep G, Chittezhath M, Kaur M, Dwyer-Nield LD, Malkinson AM and Agarwal R: Effect of silibinin on the growth and progression of primary lung tumors in mice. J Natl Cancer Inst 98: 846-855, 2006.

19. Esteller M: Non-coding RNAs in human disease. Nat Rev Genet 12: 861-874, 2011.
20. Sun M, Nie FQ, Wang ZX and De W: Involvement of lncRNA dysregulation in gastric cancer. Histol Histopathol 31: 33-39, 2016.

21. Rogoyski OM, Pueyo JI, Couso JP and Newbury SF: Functions of long non-coding RNAs in human disease and their conservation in Drosophila development. Biochem Soc Trans 45: 895-904, 2017.

22. Ruan W, Wang P, Feng S, Xue Y and Li Y: Long non-coding RNA small nucleolar RNA host gene 12 (SNHG12) promotes cell proliferation and migration by upregulating angiomotin gene expression in human osteosarcoma cells. Tumour Biol 37: 4065-4073, 2016.

23. Lan T, Ma W, Hong Z, Wu L, Chen X and Yuan Y: Long non-coding RNA small nucleolar RNA host gene 12 (SNHG12) promotes tumorigenesis and metastasis by targeting miR-199a/ b-5p in hepatocellular carcinoma. J Exp Clin Cancer Res 36: 11, 2017.

24. Wang JZ, Xu CL, Wu H and Shen SJ: LncRNA SNHG12 promotes cell growth and inhibits cell apoptosis in colorectal cancer cells. Braz J Med Biol Res 50: e6079, 2017.

25. Zhu H, Jiang X, Zhou X, Dong X, Xie K, Yang C, Jiang H, Sun X and Lu J: Neuropilin-1 regulated by miR-320 contributes to the growth and metastasis of cholangiocarcinoma cells. Liver Int: Jun 15, 2017 (Epub ahead of print).

26. Zhang T, Zou P, Wang T, Xiang J, Cheng J, Chen D and Zhou J: Down-regulation of miR-320 associated with cancer progression and cell apoptosis via targeting Mcl-1 in cervical cancer. Tumour Biol 37: 8931-8940, 2016

27. Sun JY, Xiao WZ, Wang F, Wang YQ, Zhu YH, Wu YF, Miao ZL and Lin YC: MicroRNA-320 inhibits cell proliferation in glioma by targeting E2F1. Mol Med Rep 12: 2355-2359, 2015.

28. Hsieh IS, Chang KC, Tsai YT, Ke JY, Lu PJ, Lee KH, Yeh SD, Hong TM and Chen YL: MicroRNA-320 suppresses the stem cell-like characteristics of prostate cancer cells by downregulating the Wnt/beta-catenin signaling pathway. Carcinogenesis 34 : $530-538,2013$

29. Wang W, Yang J, Xiang YY, Pi J and Bian J: Overexpression of Hsa-miR-320 Is associated with invasion and metastasis of ovarian cancer. J Cell Biochem 118: 3654-3661, 2017.

30. Zhao Y, Dong Q and Wang E: MicroRNA-320 inhibits invasion and induces apoptosis by targeting CRKL and inhibiting ERK and AKT signaling in gastric cancer cells. Onco Targets Ther 10: 1049-1058, 2017. 